\title{
Estimating Effective Steric and Electronic Impacts of Ferrocenyl Group in Organophosphines
}

\author{
Hao Hu ${ }^{\dagger}, \stackrel{+}{ }$ Hiroki Ichiryu,${ }^{\dagger}$ Kiyohiko Nakajima, ${ }^{\S}$ and Masamichi Ogasawara ${ }^{*}, \dagger$ \\ 'Department of Natural Science, Graduate School of Science and Technology, Tokushima International \\ Science Institute, and Research Cluster on "Innovative Chemical Sensing", Tokushima University, \\ Tokushima 770-8506, Japan. ${ }^{\ddagger}$ Graduate School of Life Science, Hokkaido University, Kita-ku, Sapporo \\ 001-0021, Japan. \$Department of Chemistry, Aichi University of Education, Igaya, Kariya, Aichi 448- \\ 8542, Japan.
}

\section{Supporting Information}

\section{Table of Contents}

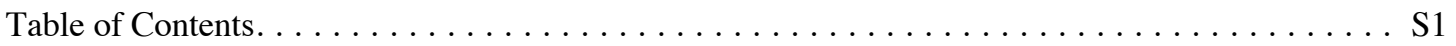

${ }^{1} \mathrm{H}-,{ }^{13} \mathrm{C}\left\{{ }^{1} \mathrm{H}\right\}-$, and ${ }^{31} \mathrm{P}\left\{{ }^{1} \mathrm{H}\right\}$-NMR Spectra of Monodentate Phosphines 1a-d. . . . . . . . . S2-S13

${ }^{1} \mathrm{H}-,{ }^{13} \mathrm{C}\left\{{ }^{1} \mathrm{H}\right\}-$, and ${ }^{31} \mathrm{P}\left\{{ }^{1} \mathrm{H}\right\}-N M R$ Spectra of (Phosphine) $\mathrm{Au}(\mathrm{I}) \mathrm{Cl}$ Complexes 13a-c. . . . . . . . S14-S22

FT-IR Spectra of $\mathrm{Ni}(\mathrm{CO})_{3}\left(\mathrm{Phosphine)}\right.$ Complexes 2a-d in $\mathrm{CH}_{2} \mathrm{Cl}_{2} \ldots \ldots \ldots \ldots \ldots \ldots \ldots$. $\ldots \ldots \ldots$ 


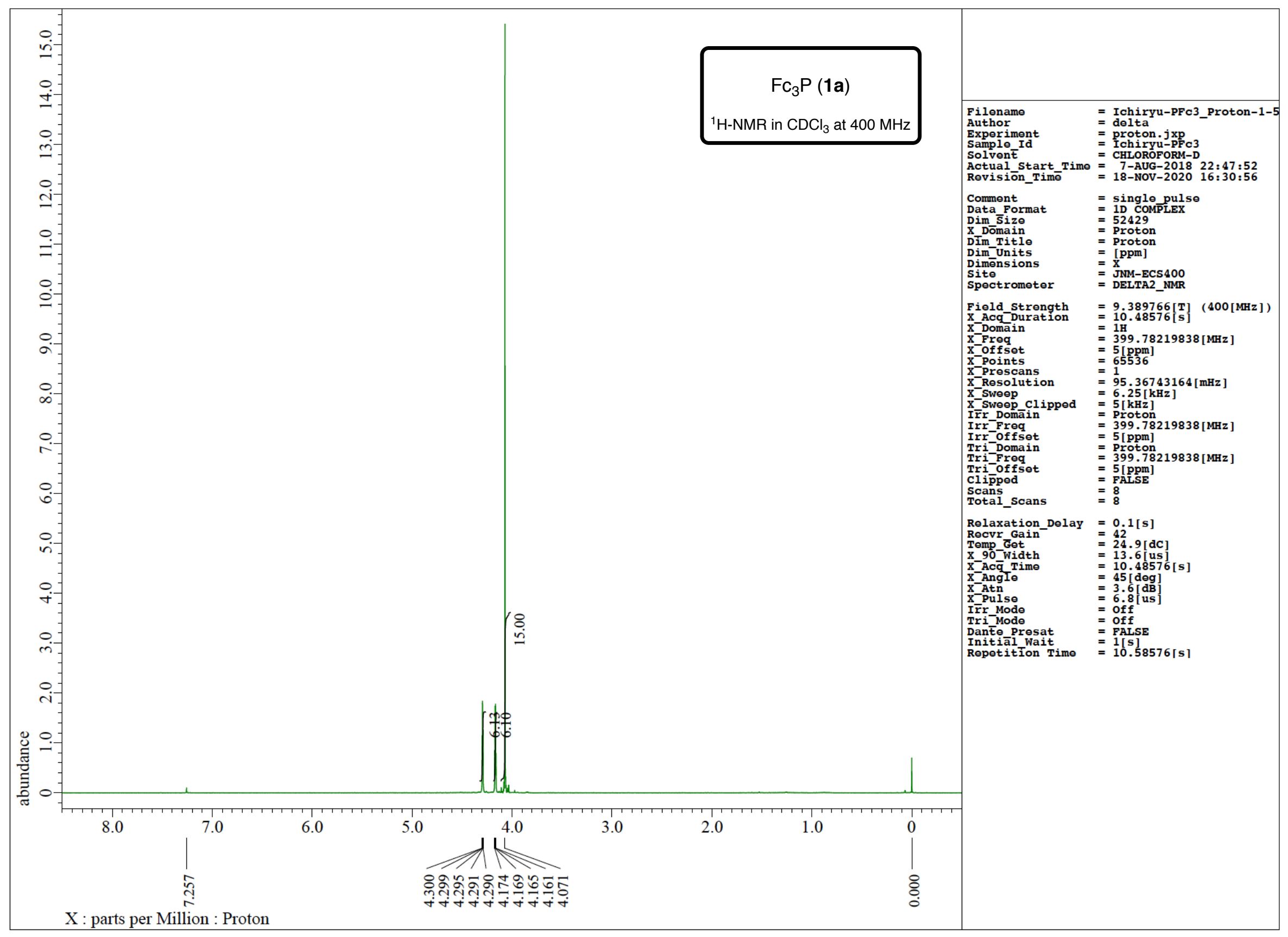

Figure S1. ${ }^{1} \mathrm{H}-\mathrm{NMR}$ Spectrum of Triferrocenylphosphine (1a) in $\mathrm{CDCl}_{3}$ at $400 \mathrm{MHz}$. 


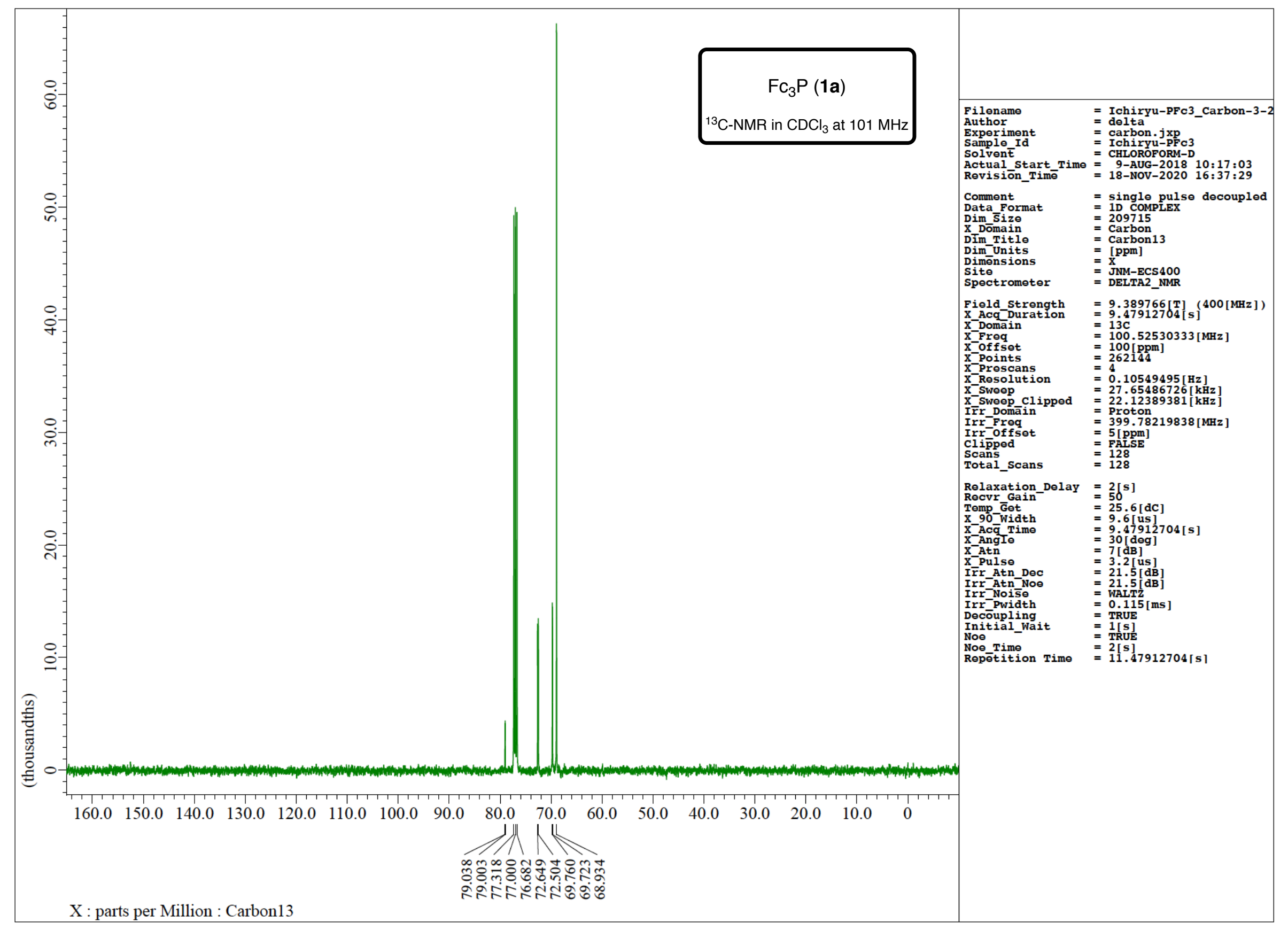

Figure S2. ${ }^{13} \mathrm{C}-\mathrm{NMR}$ Spectrum of Triferrocenylphosphine (1a) in $\mathrm{CDCl}_{3}$ at $101 \mathrm{MHz}$. 


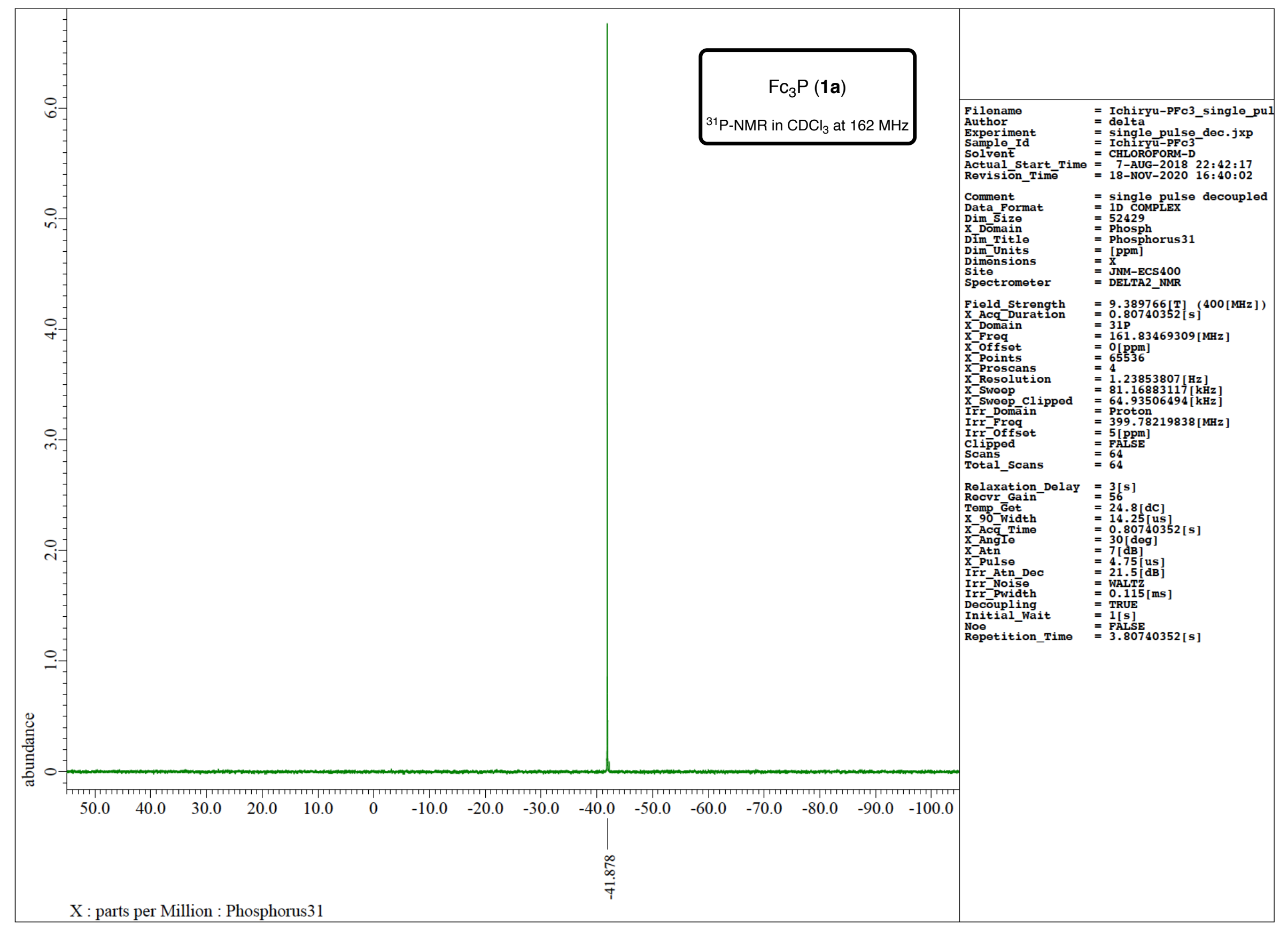

Figure S3. ${ }^{31} \mathrm{P}-\mathrm{NMR}$ Spectrum of Triferrocenylphosphine (1a) in $\mathrm{CDCl}_{3}$ at $162 \mathrm{MHz}$. 


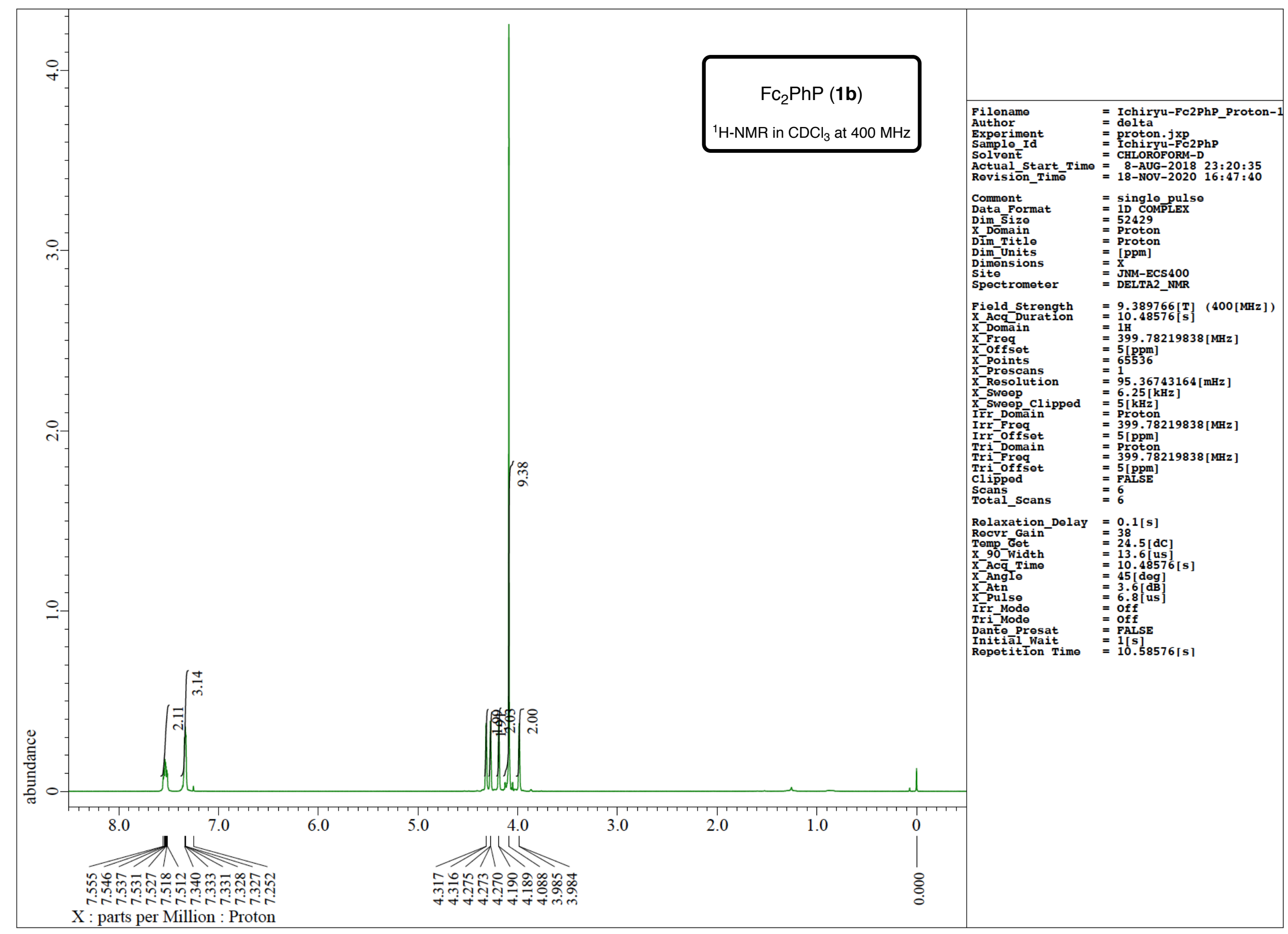

Figure S4. ${ }^{1} \mathrm{H}-\mathrm{NMR}$ Spectrum of Diferrocenylphenylphosphine (1b) in $\mathrm{CDCl}_{3}$ at $400 \mathrm{MHz}$. 


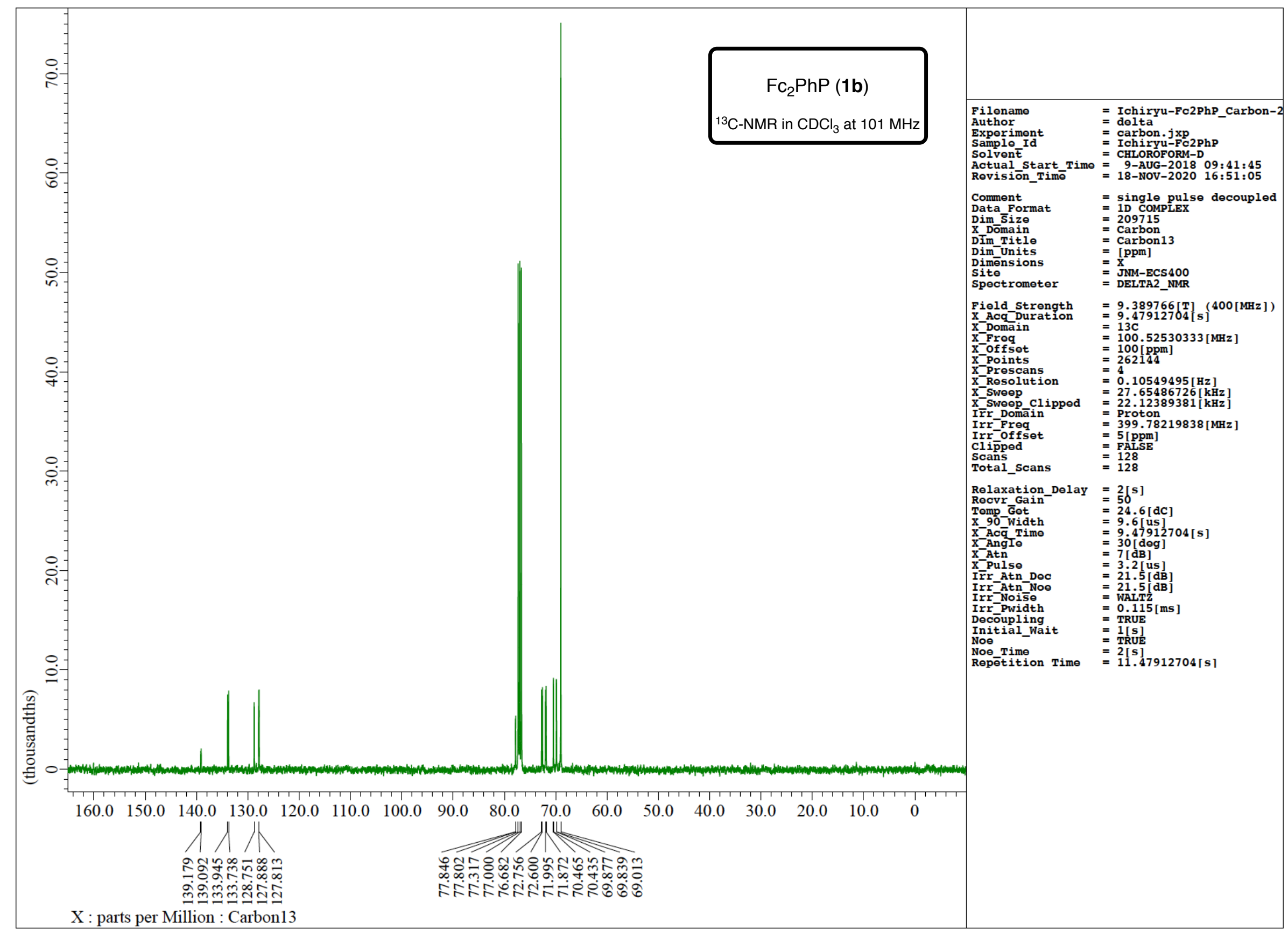

Figure S5. ${ }^{13} \mathrm{C}-\mathrm{NMR}$ Spectrum of Diferrocenylphenylphosphine (1b) in $\mathrm{CDCl}_{3}$ at $101 \mathrm{MHz}$. 


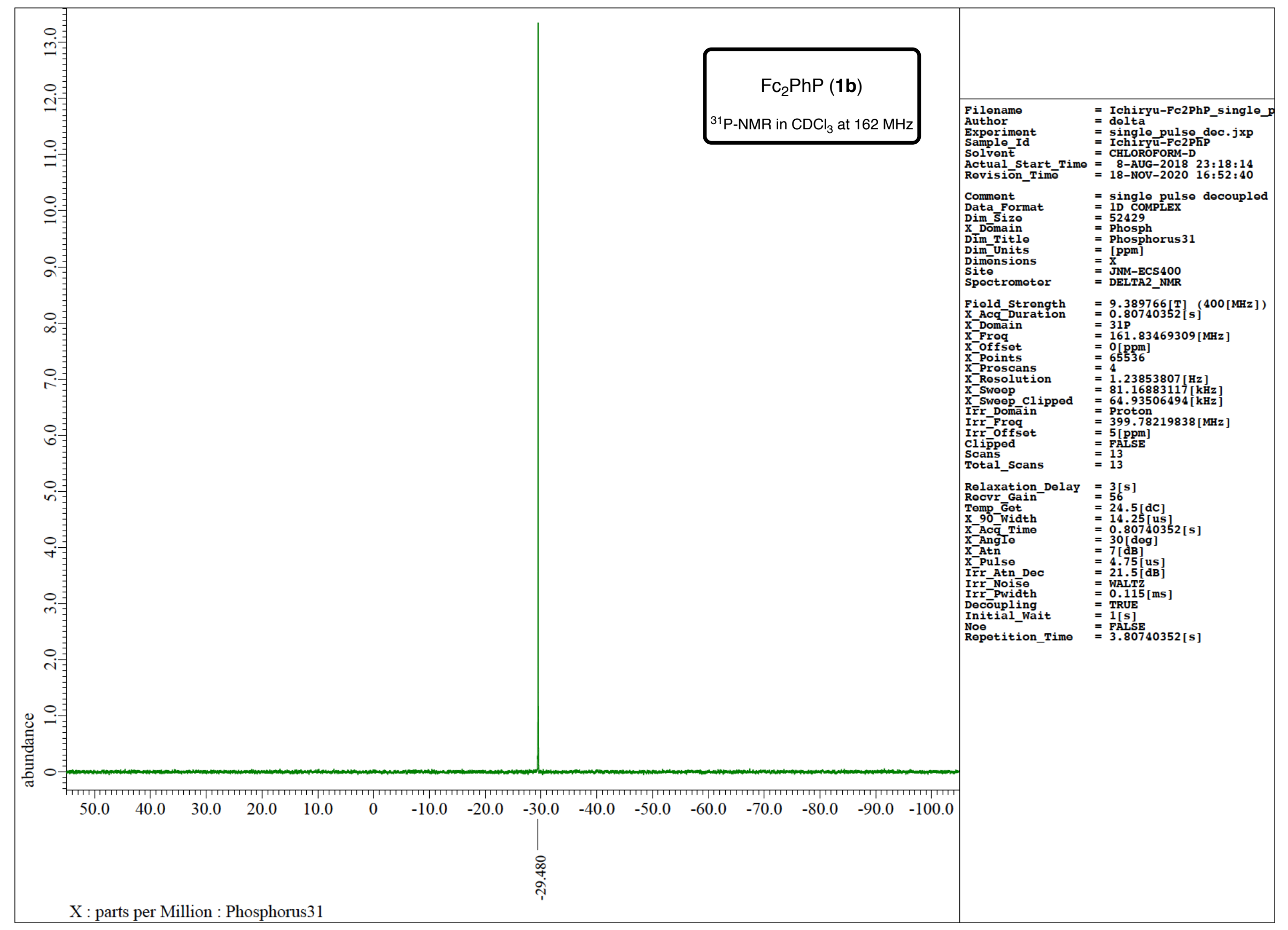

Figure S6. ${ }^{31} \mathrm{P}-\mathrm{NMR}$ Spectrum of Diferrocenylphenylphosphine (1b) in $\mathrm{CDCl}_{3}$ at $162 \mathrm{MHz}$. 


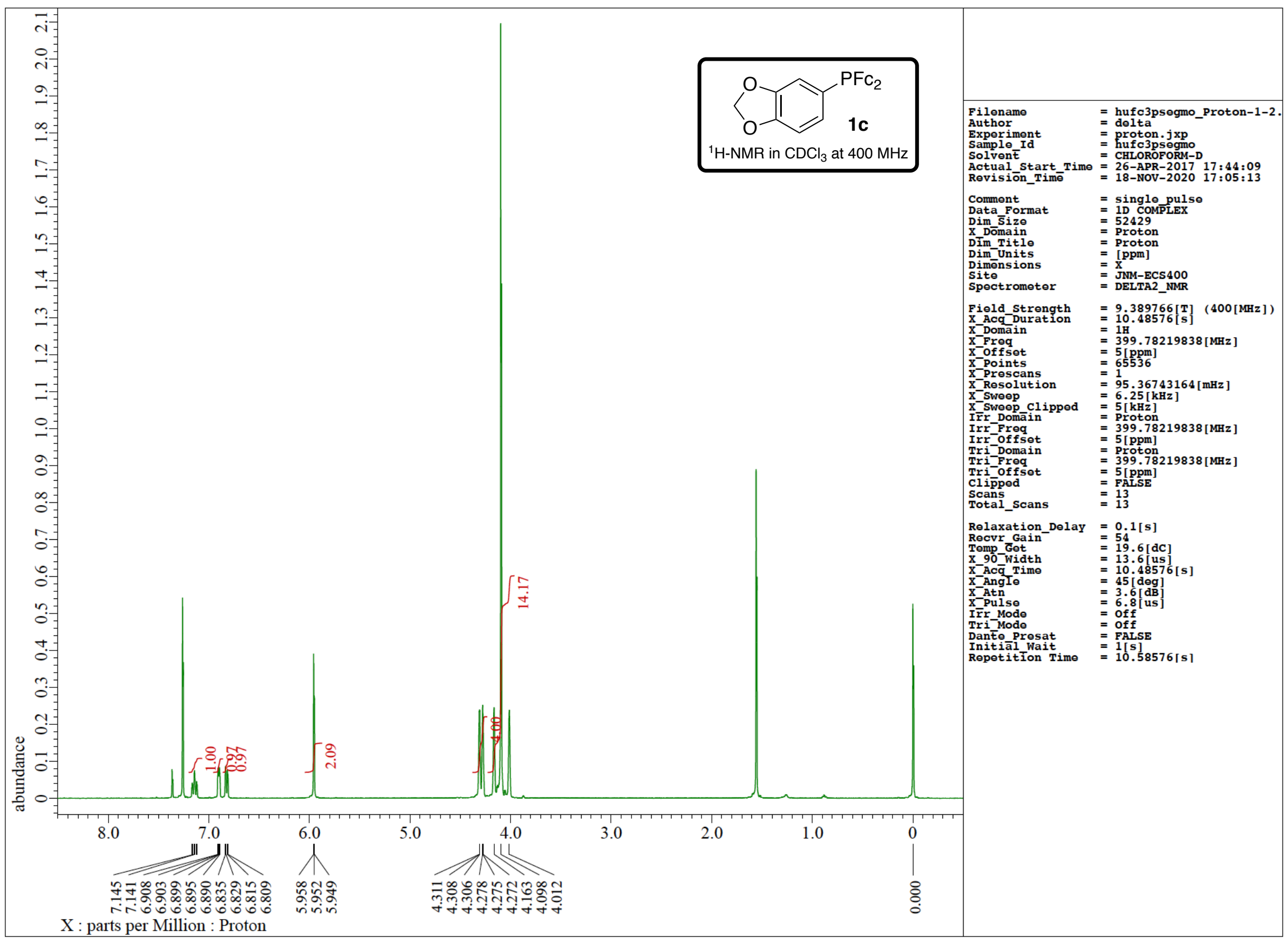

Figure S7. ${ }^{1} \mathrm{H}-\mathrm{NMR}$ Spectrum of 1,3-Benzodioxol-5-yldiferrocenylphosphine (1c) in $\mathrm{CDCl}_{3}$ at $400 \mathrm{MHz}$. 


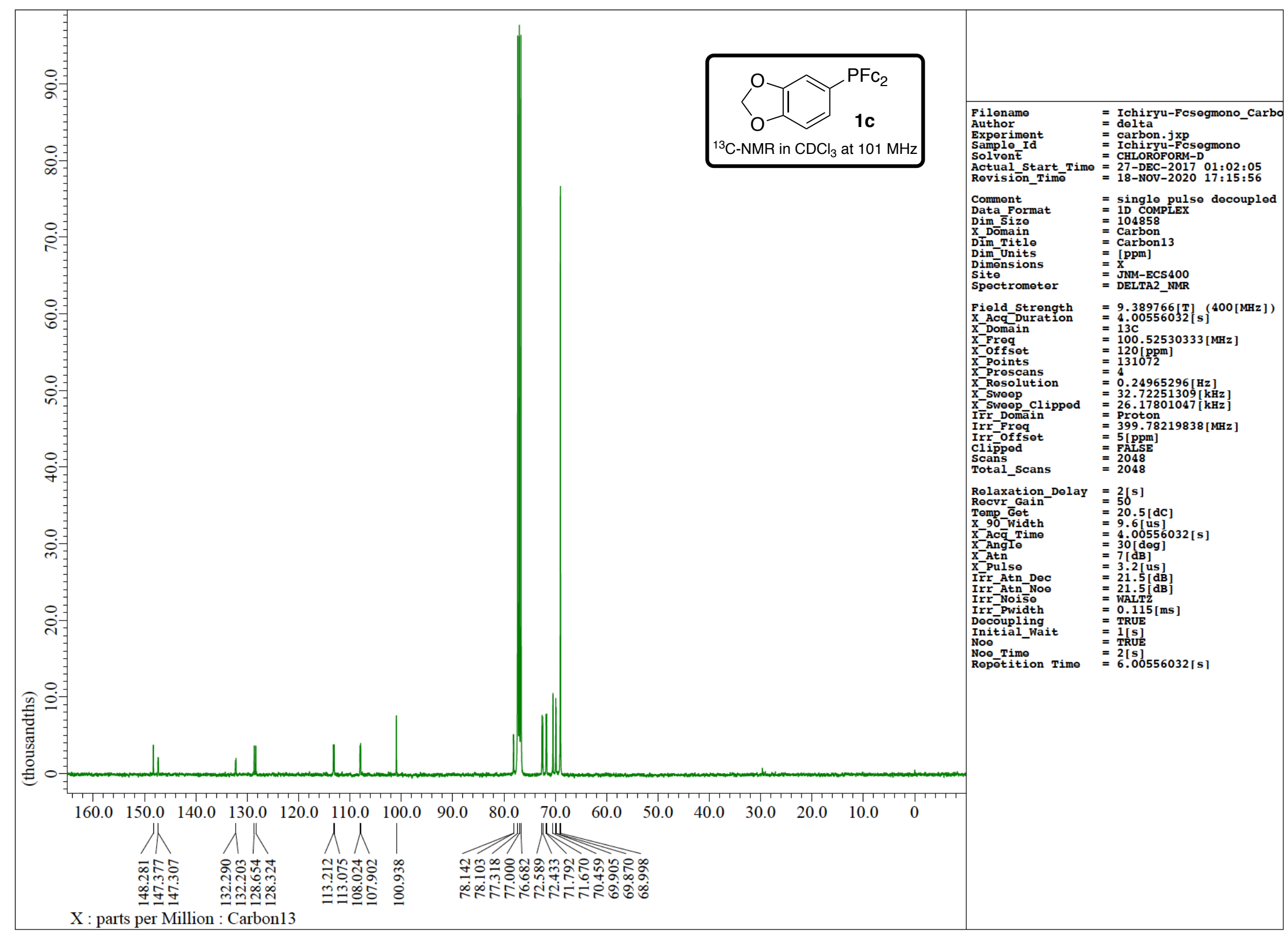

Figure S8. ${ }^{13} \mathrm{C}-\mathrm{NMR}$ Spectrum of 1,3-Benzodioxol-5-yldiferrocenylphosphine (1c) in $\mathrm{CDCl}_{3}$ at $101 \mathrm{MHz}$. 


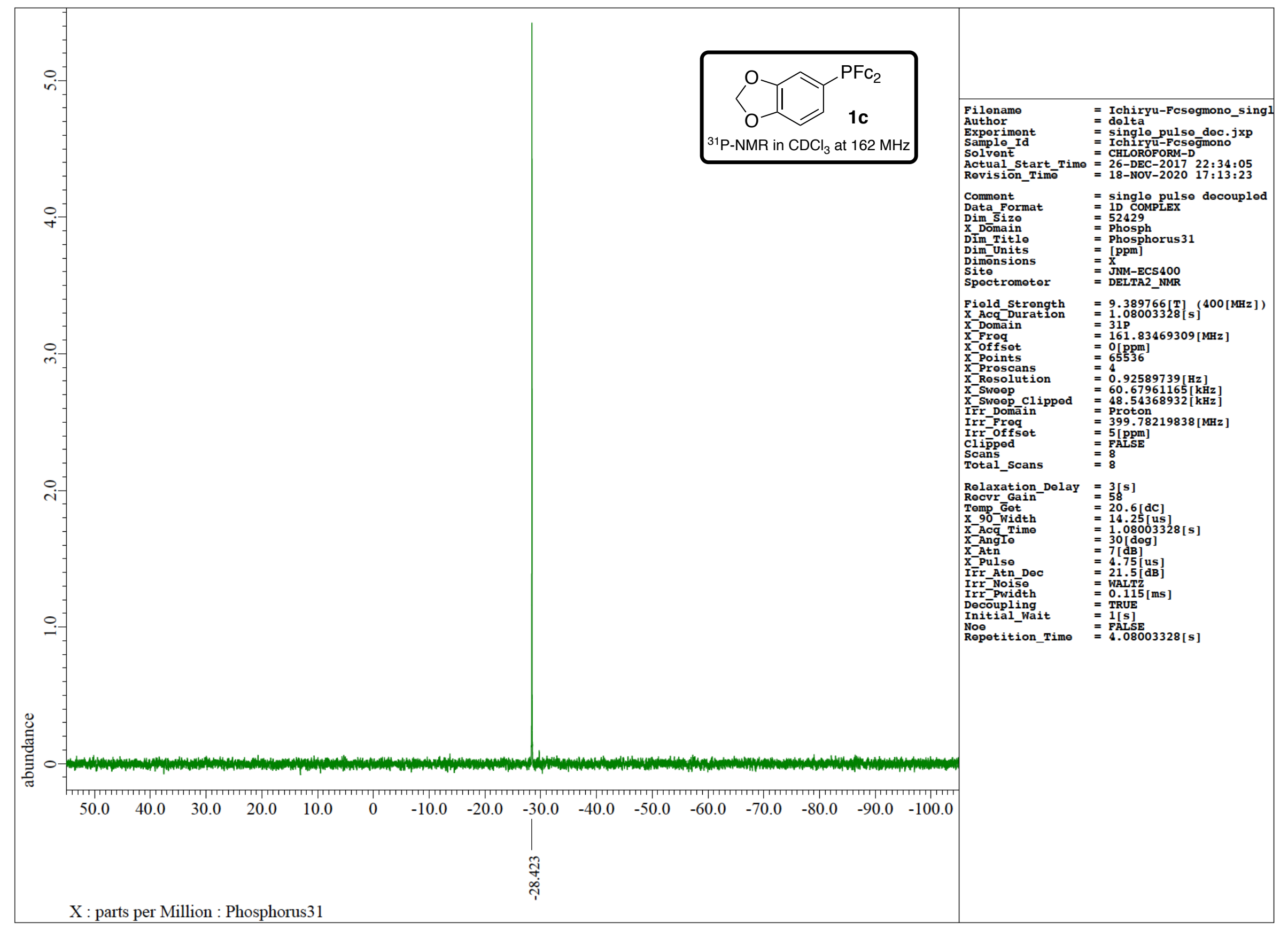

Figure S9. ${ }^{31} \mathrm{P}-\mathrm{NMR}$ Spectrum of 1,3-Benzodioxol-5-yldiferrocenylphosphine (1c) in $\mathrm{CDCl}_{3}$ at $162 \mathrm{MHz}$.

-S10- 


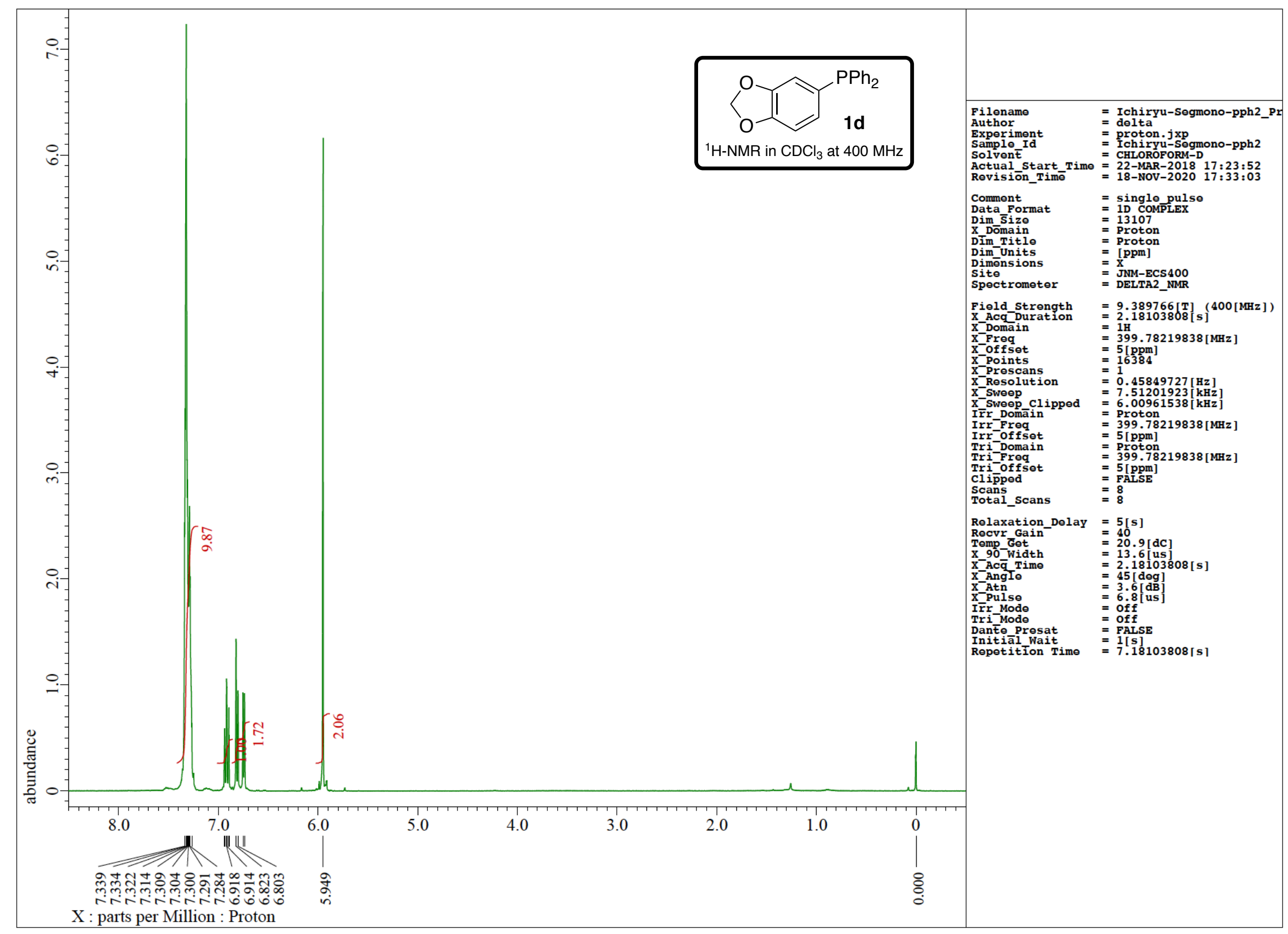

Figure S10. ${ }^{1} \mathrm{H}-\mathrm{NMR}$ Spectrum of 1,3-Benzodioxol-5-yldiphenylphosphine (1d) in $\mathrm{CDCl}_{3}$ at $400 \mathrm{MHz}$. 


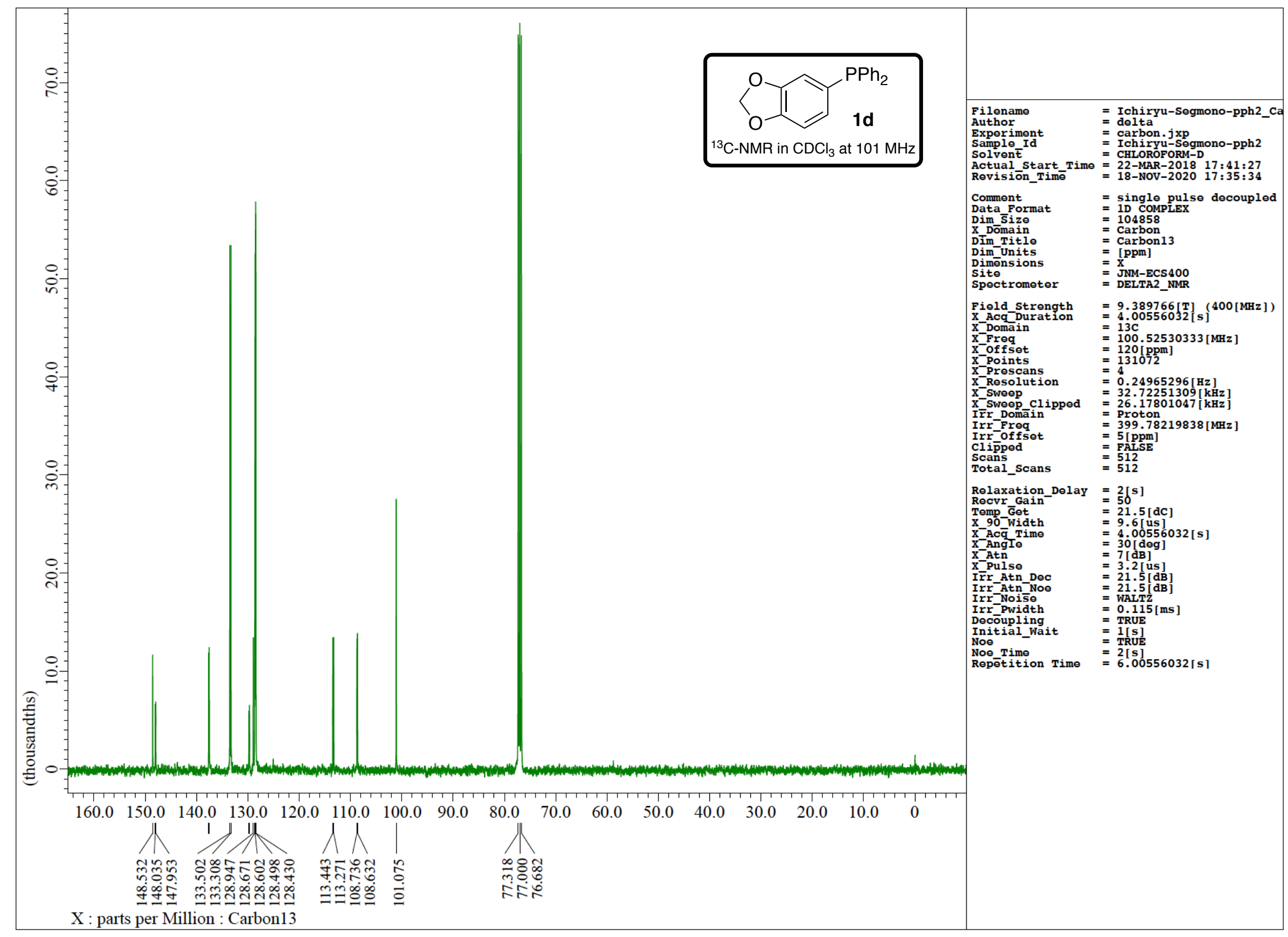

Figure S11. ${ }^{13} \mathrm{C}$-NMR Spectrum of 1,3-Benzodioxol-5-yldiphenylphosphine (1d) in $\mathrm{CDCl}_{3}$ at $101 \mathrm{MHz}$.

-S12- 


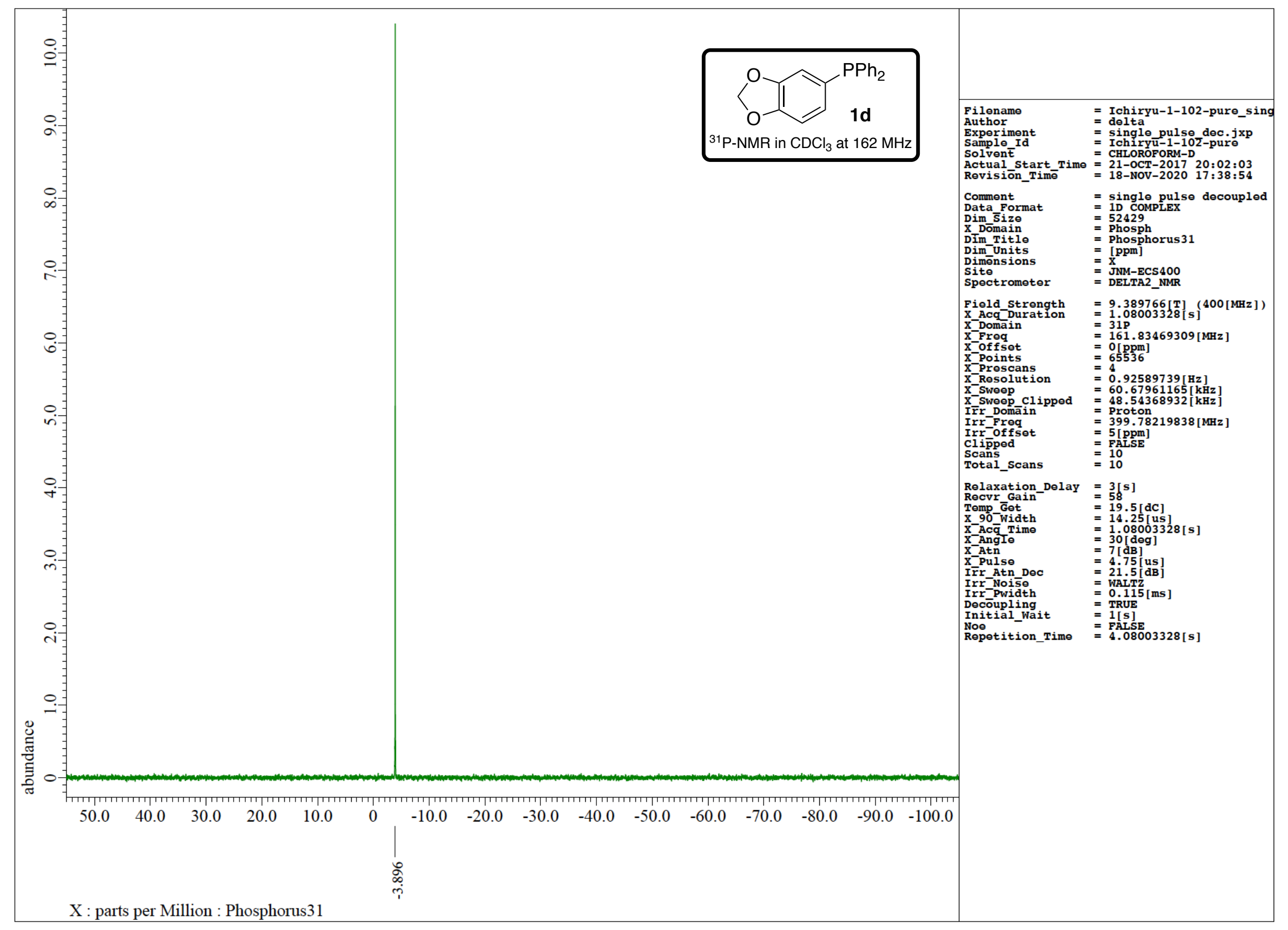

Figure S12. ${ }^{31} \mathrm{P}-\mathrm{NMR}$ Spectrum of 1,3-Benzodioxol-5-yldiphenylphosphine (1d) in $\mathrm{CDCl}_{3}$ at $162 \mathrm{MHz}$. 


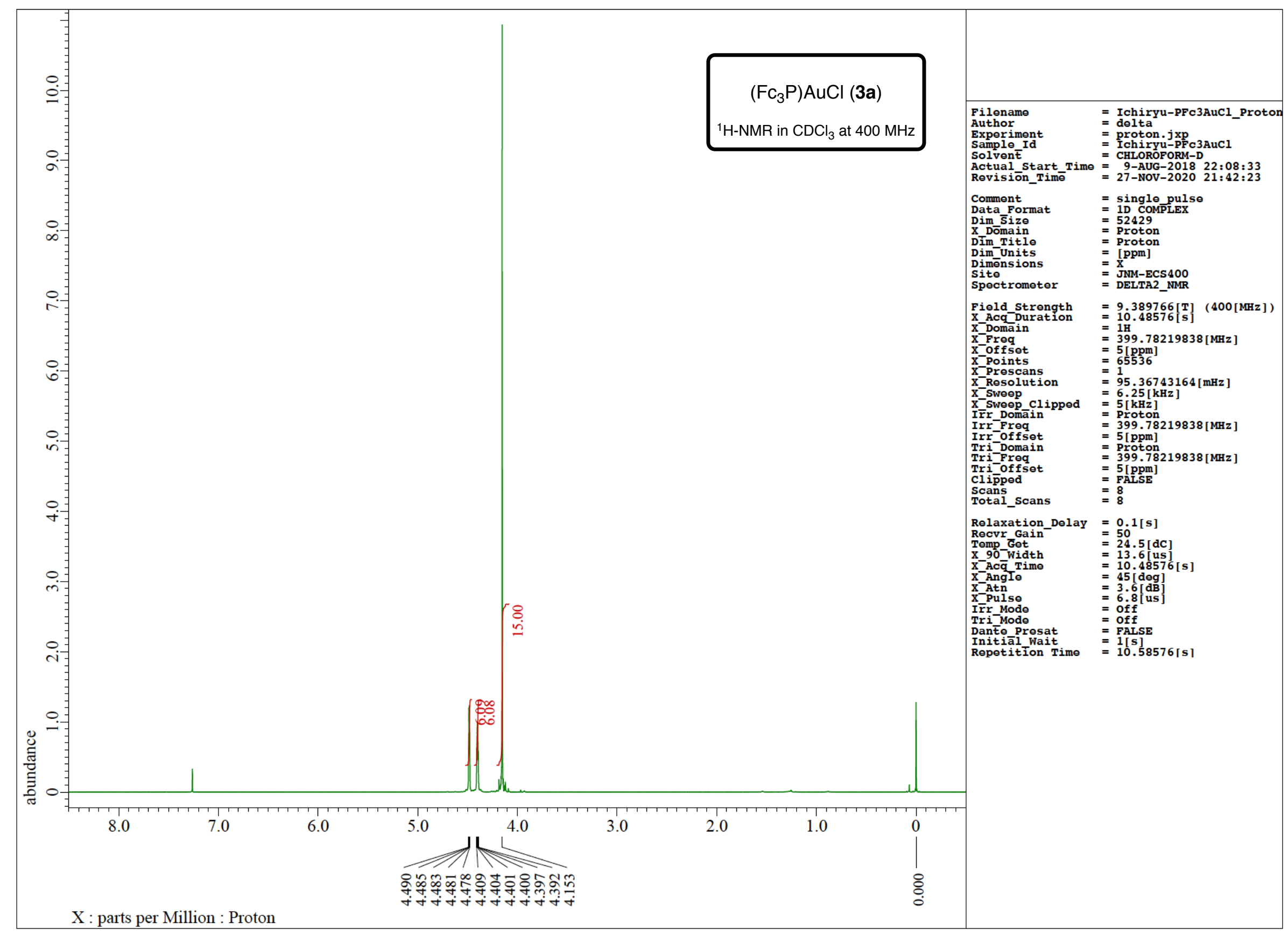

Figure S13. ${ }^{1} \mathrm{H}-\mathrm{NMR}$ Spectrum of Chloro(triferrocenylphosphine)gold(I) (3a) in $\mathrm{CDCl}_{3}$ at $400 \mathrm{MHz}$. 


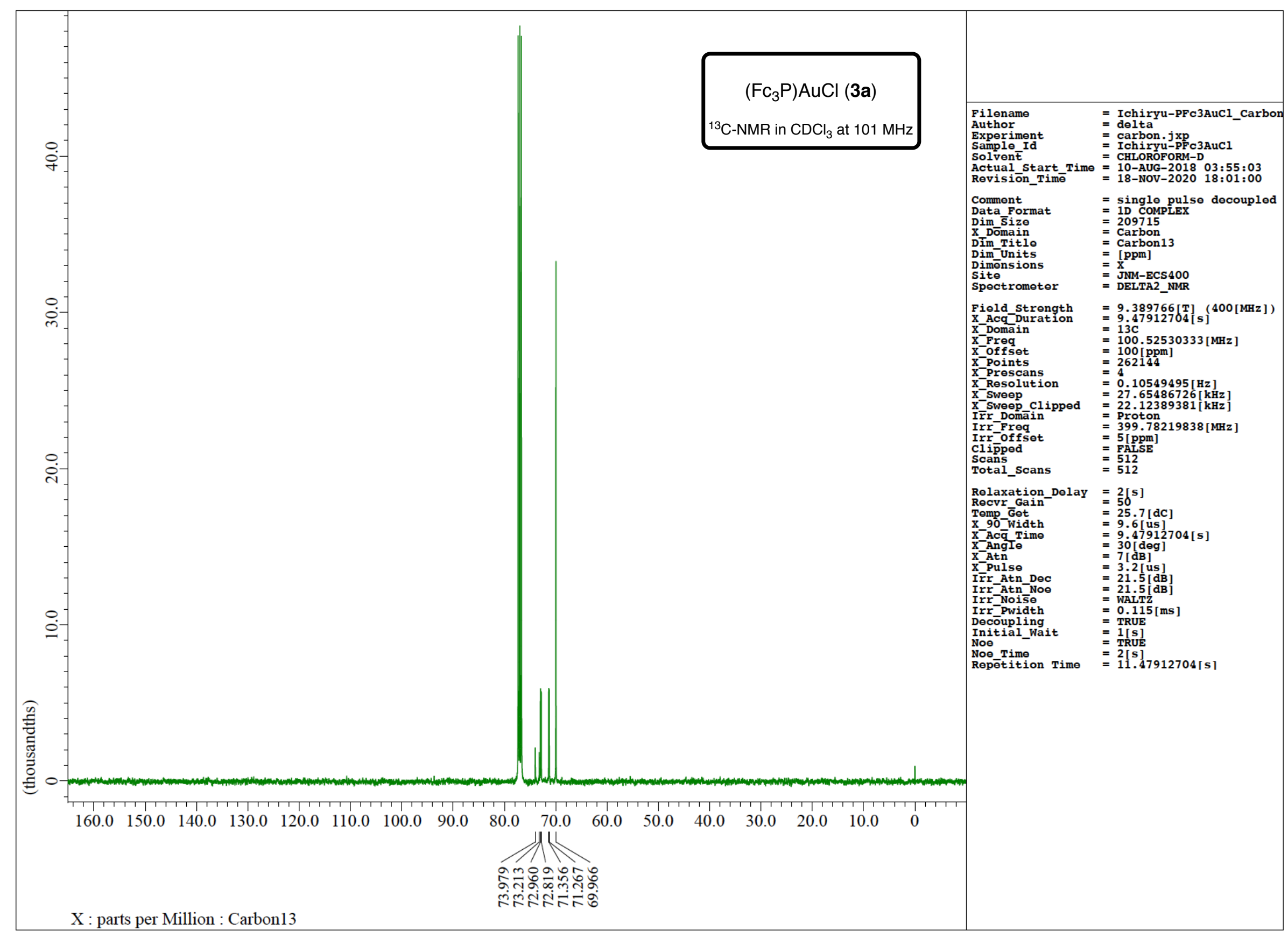

Figure S14. ${ }^{13} \mathrm{C}-\mathrm{NMR}$ Spectrum of Chloro(triferrocenylphosphine)gold(I) (3a) in $\mathrm{CDCl}_{3}$ at $101 \mathrm{MHz}$. 


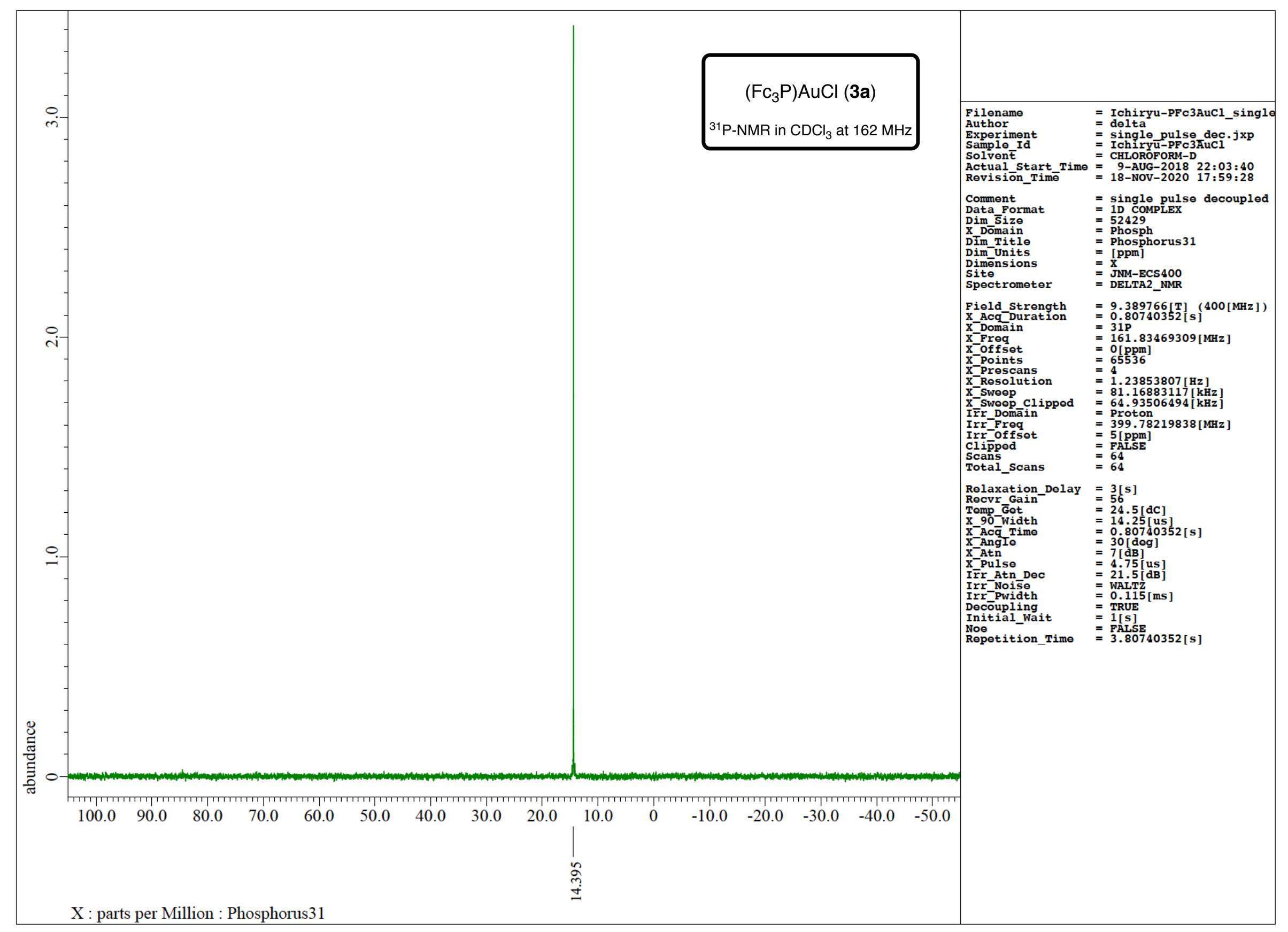

Figure S15. ${ }^{31} \mathrm{P}-\mathrm{NMR}$ Spectrum of Chloro(triferrocenylphosphine)gold(I) (3a) in $\mathrm{CDCl}_{3}$ at $162 \mathrm{MHz}$. 


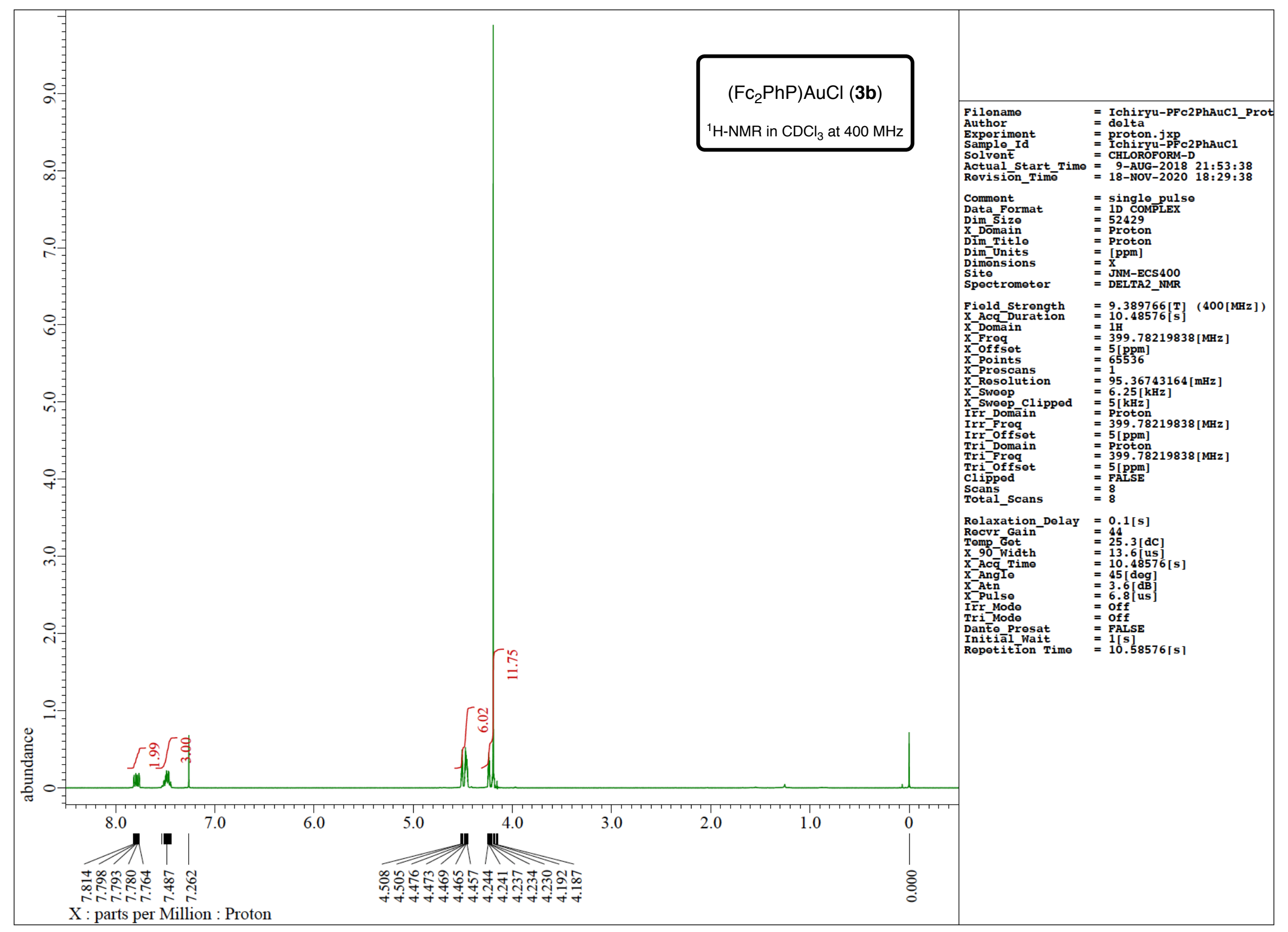

Figure S16. ${ }^{1} \mathrm{H}-\mathrm{NMR}$ Spectrum of Chloro(diferrocenylphenylphosphine)gold(I) (3b) in $\mathrm{CDCl}_{3}$ at $400 \mathrm{MHz}$. 


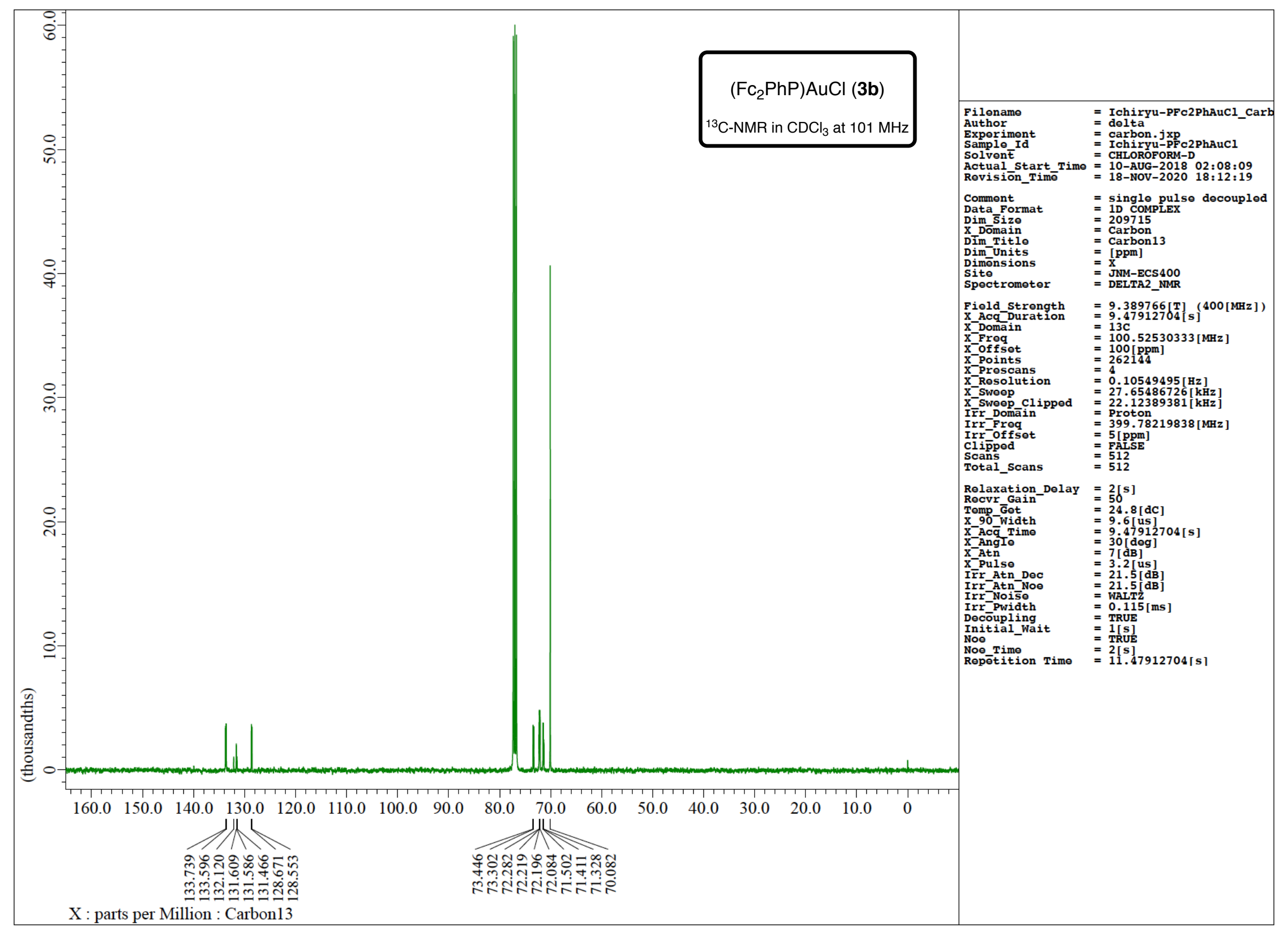

Figure S17. ${ }^{13} \mathrm{C}-\mathrm{NMR}$ Spectrum of Chloro(diferrocenylphenylphosphine)gold(I) (3b) in $\mathrm{CDCl}_{3}$ at $101 \mathrm{MHz}$. 


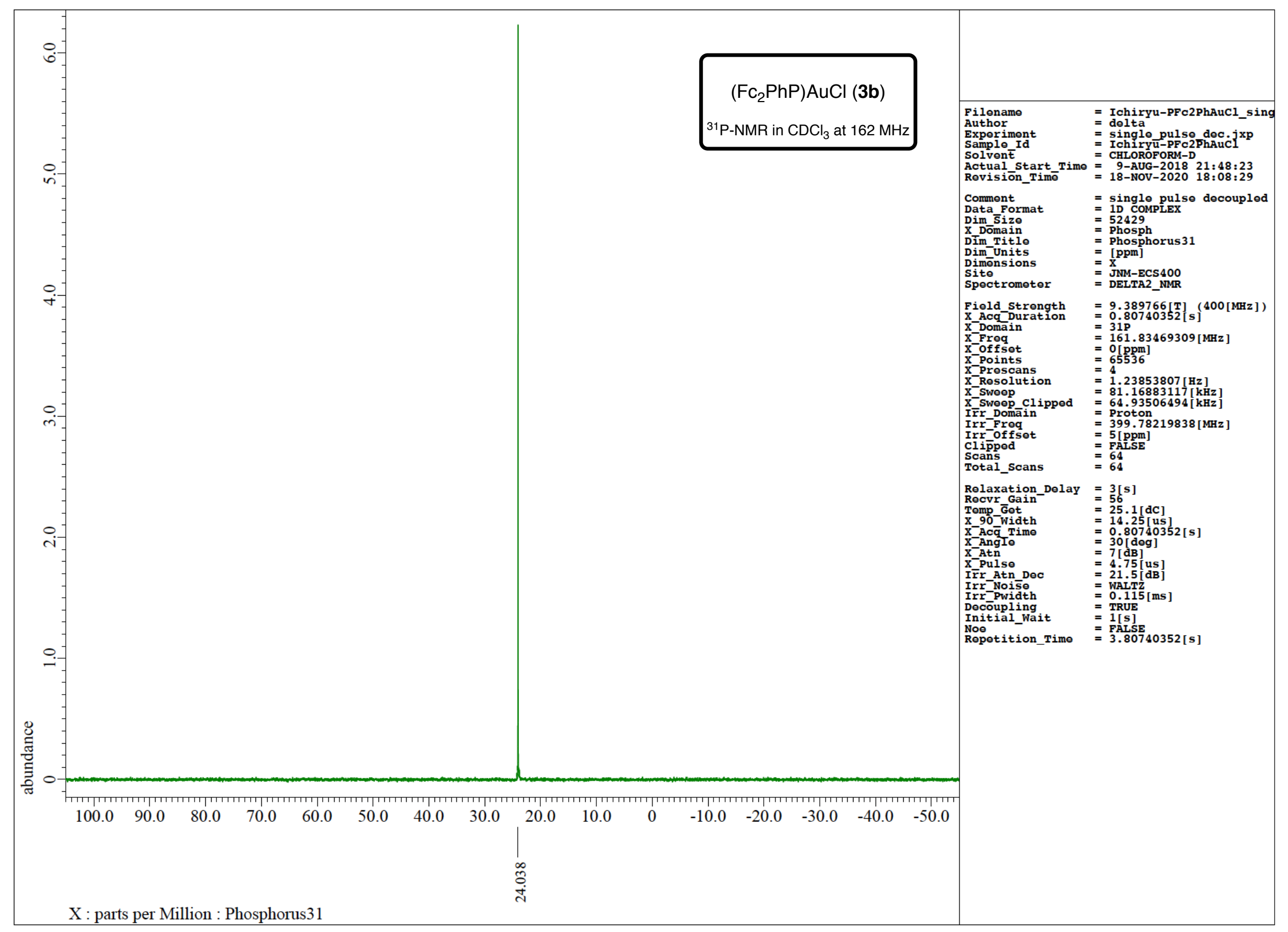

Figure S18. ${ }^{31} \mathrm{P}-\mathrm{NMR}$ Spectrum of Chloro(diferrocenylphenylphosphine)gold(I) (3b) in $\mathrm{CDCl}_{3}$ at $162 \mathrm{MHz}$. 


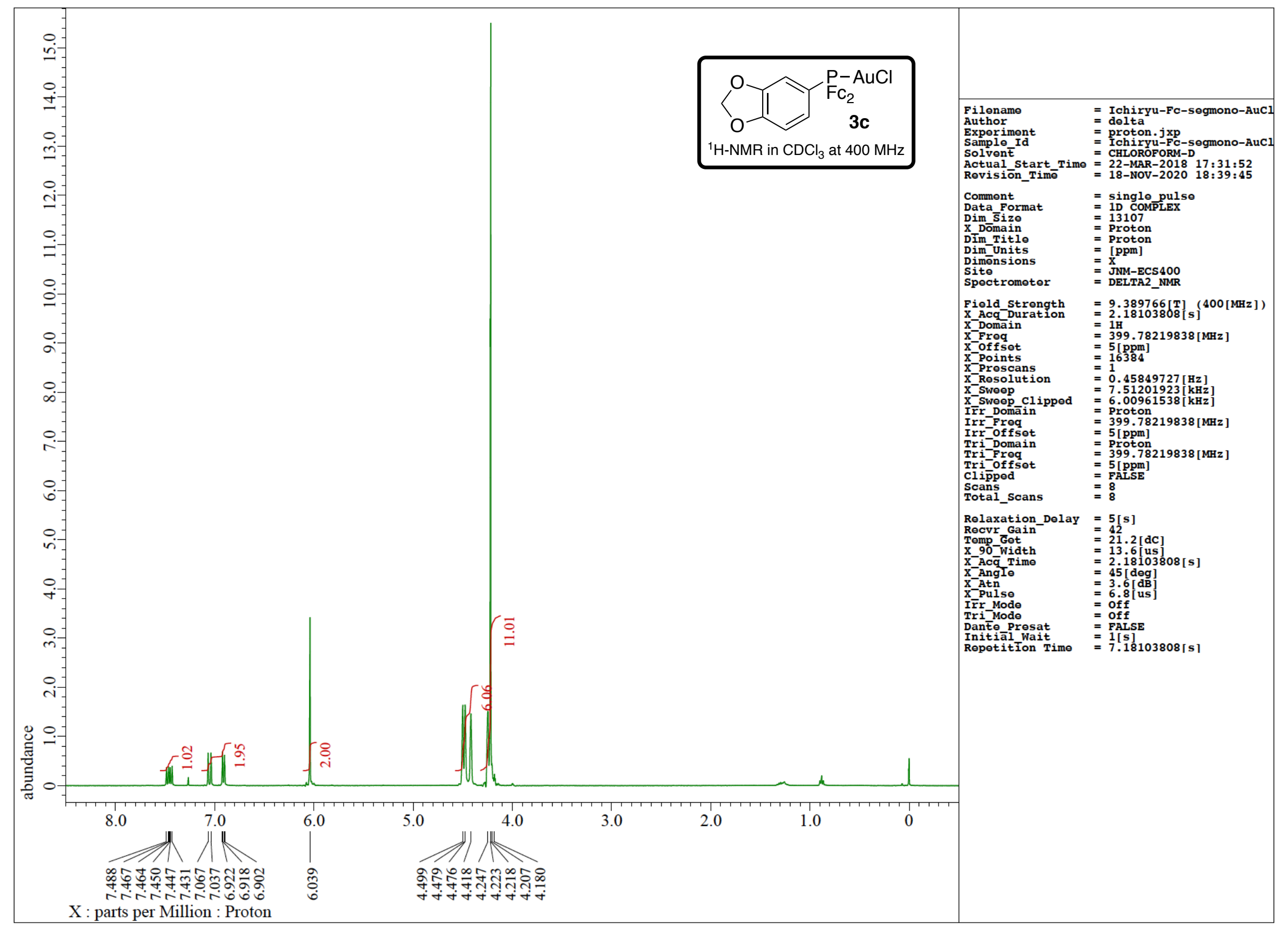

Figure S19. ${ }^{1} \mathrm{H}-\mathrm{NMR}$ Spectrum of Chloro(1,3-benzodioxol-5-yldiferrocenylphosphine)gold(I) (3c) in $\mathrm{CDCl}_{3}$ at $400 \mathrm{MHz}$. 


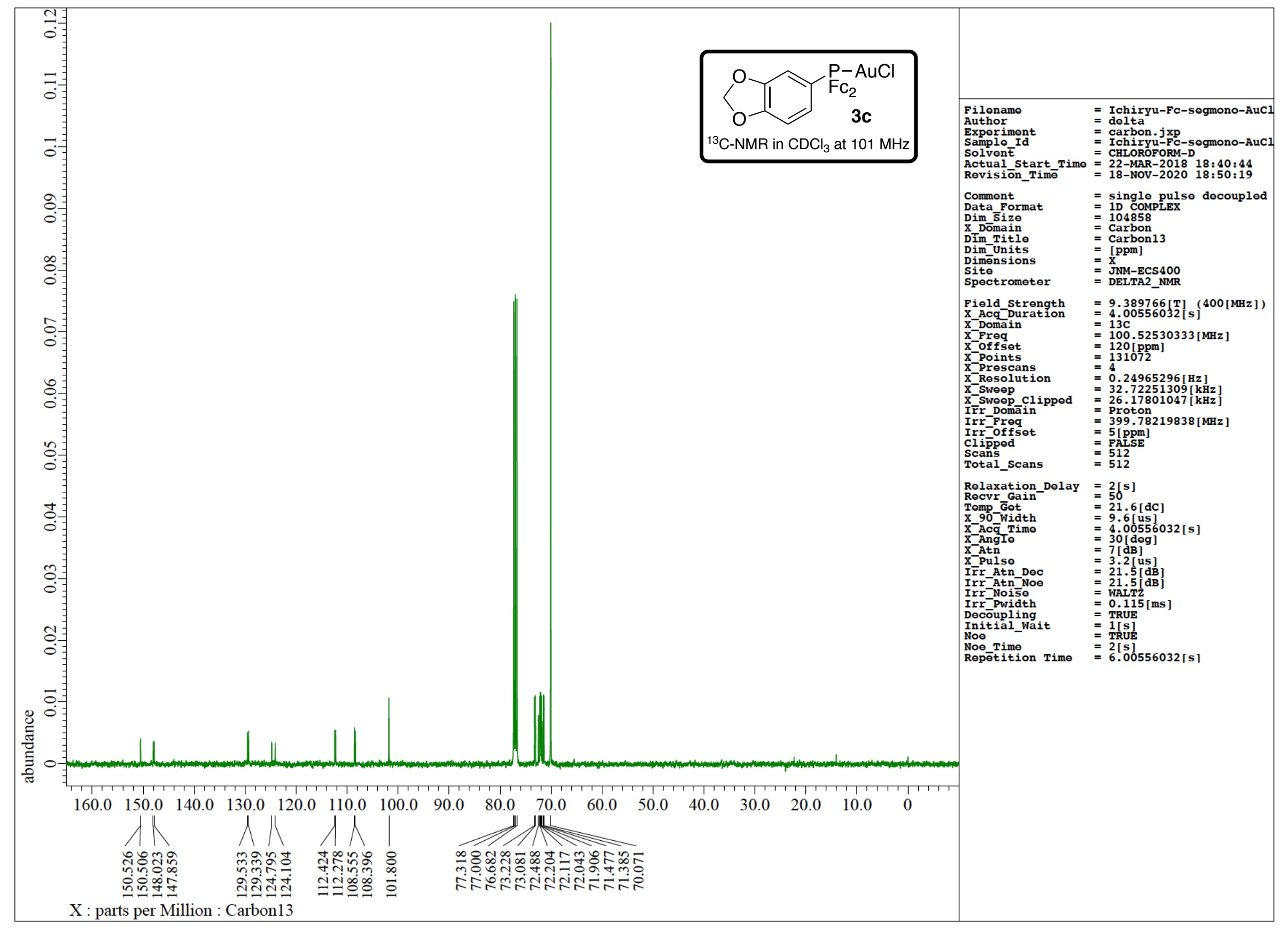

Figure S20. ${ }^{13} \mathrm{C}-\mathrm{NMR}$ Spectrum of Chloro(1,3-benzodioxol-5-yldiferrocenylphosphine)gold(I) (3c) in $\mathrm{CDCl}_{3}$ at $101 \mathrm{MHz}$. 


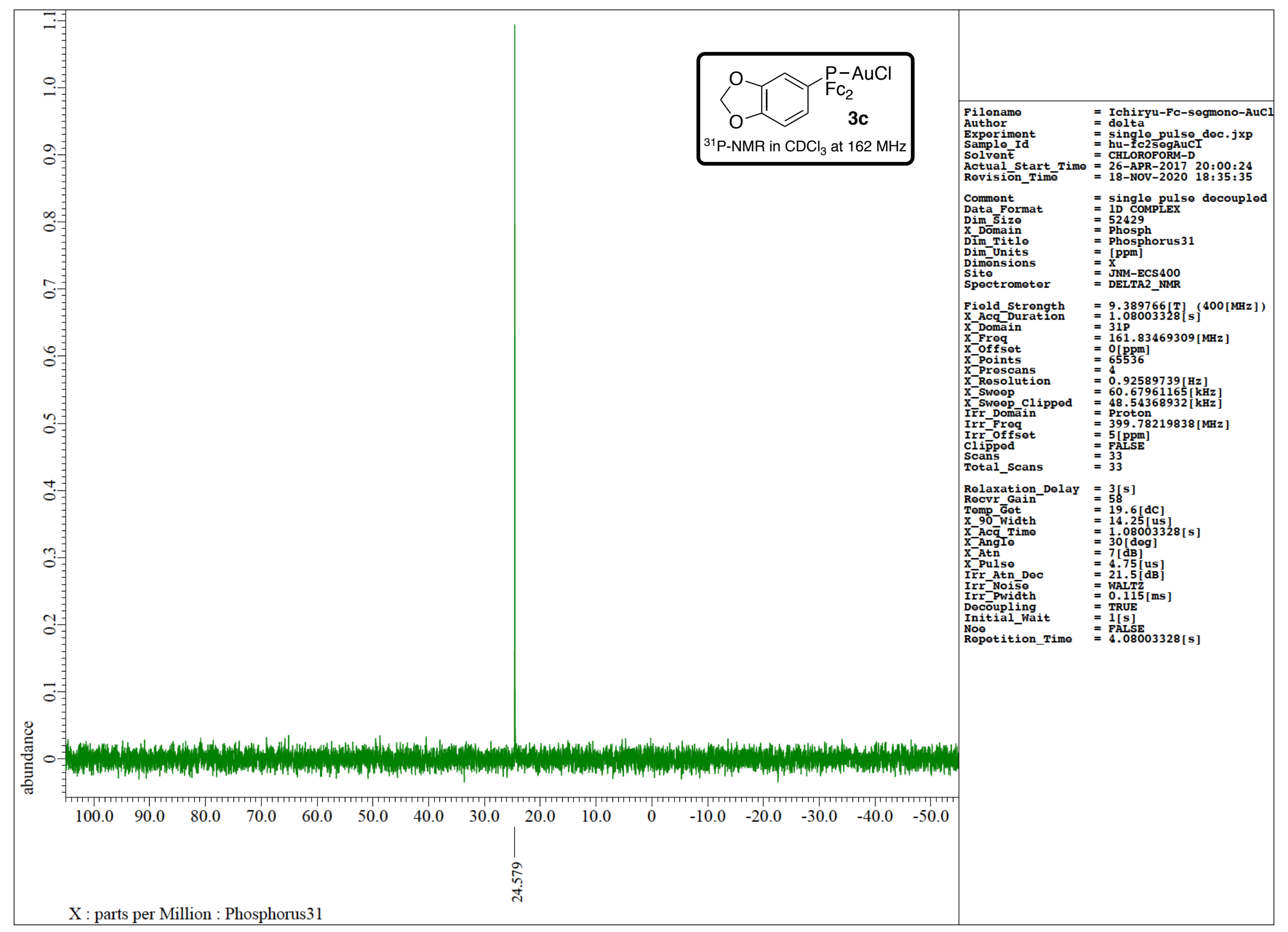

Figure S21. ${ }^{31} \mathrm{P}-\mathrm{NMR}$ Spectrum of Chloro(1,3-benzodioxol-5-yldiferrocenylphosphine)gold(I) (3c) in $\mathrm{CDCl}_{3}$ at $162 \mathrm{MHz}$. 


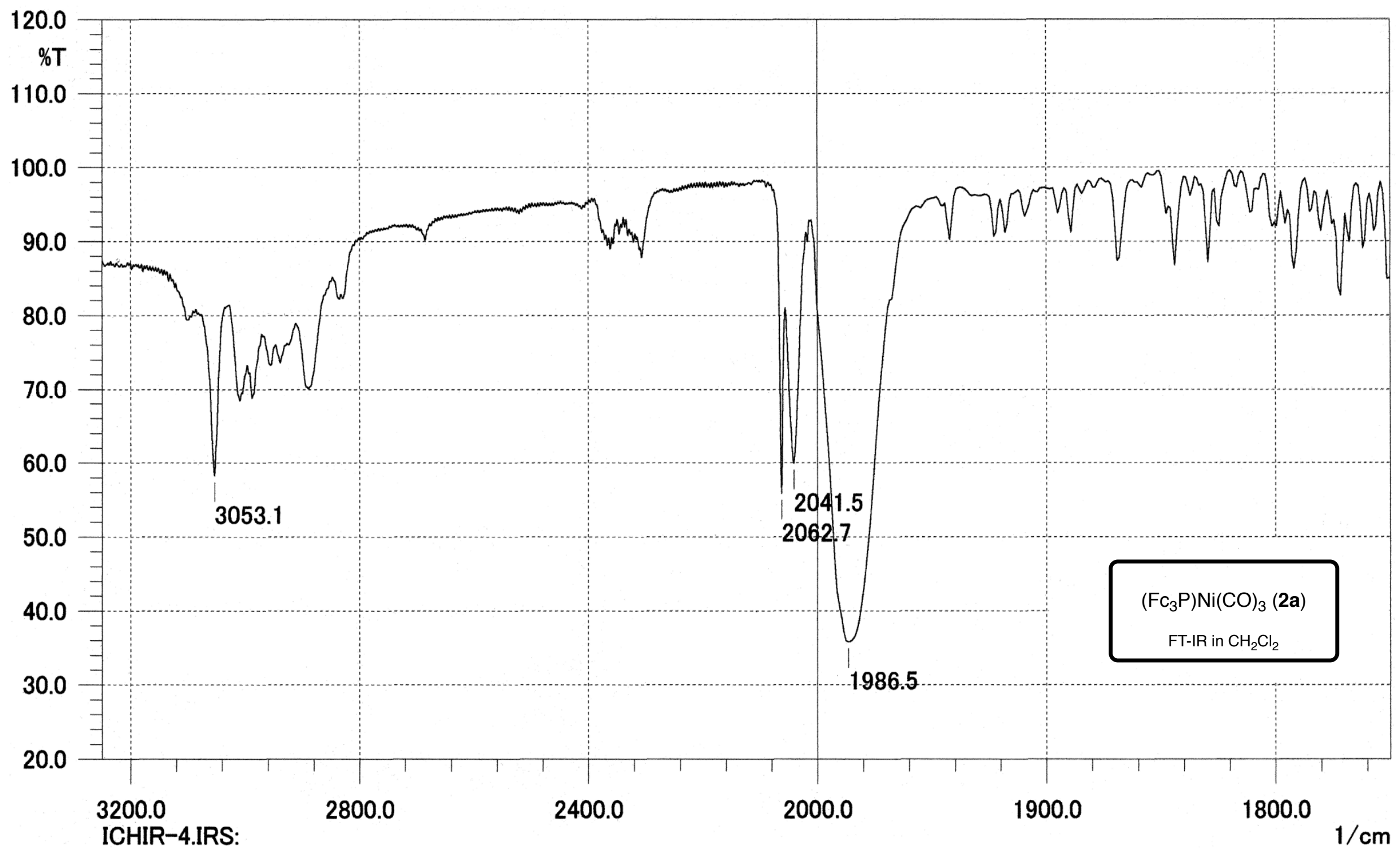

Figure S22. FT-IR Spectrum of $\mathrm{Ni}(\mathrm{CO})_{3}\left(\mathrm{PFc}_{3}\right)(\mathbf{2 a})$ in $\mathrm{CH}_{2} \mathrm{Cl}_{2}$. 


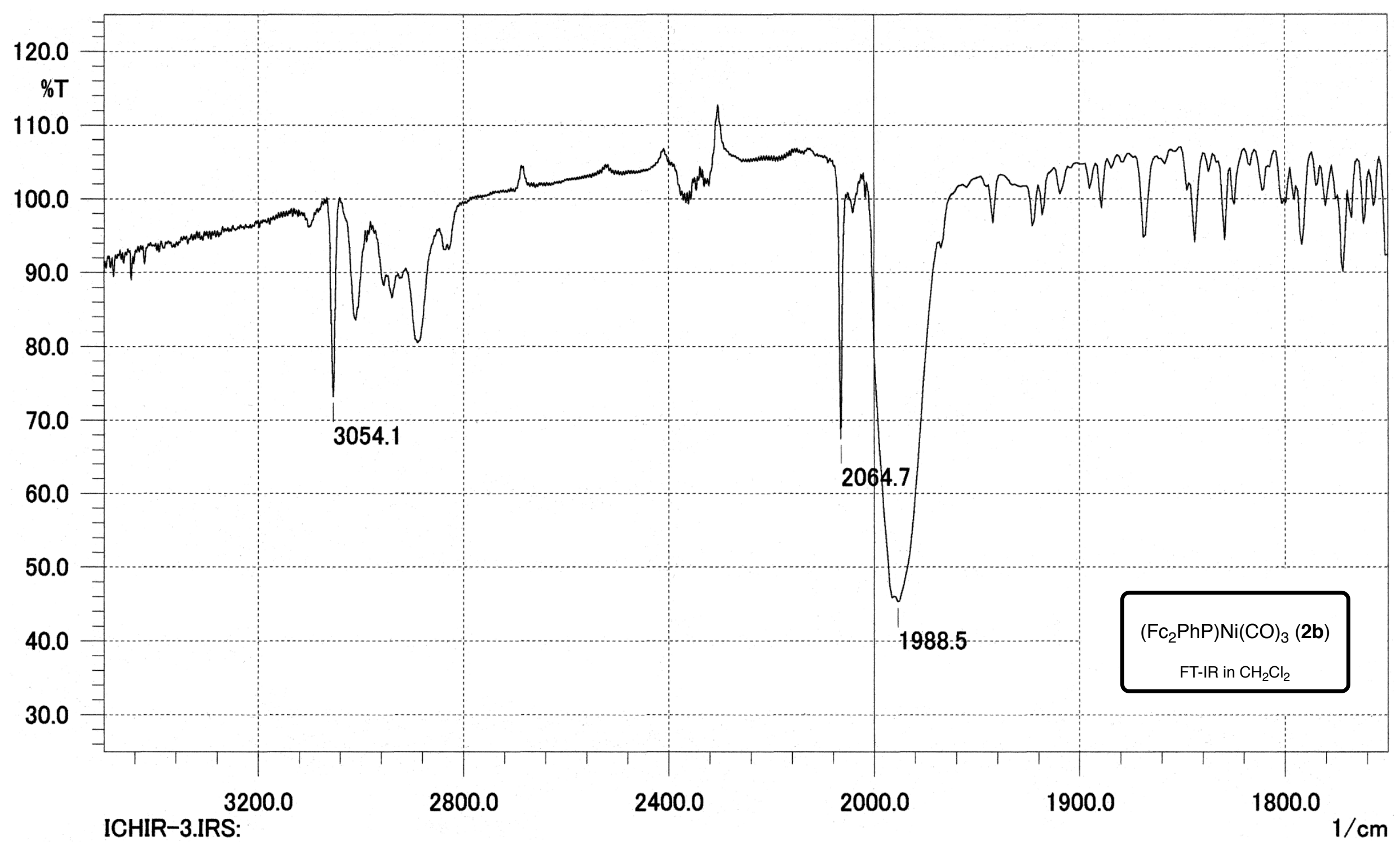

Figure S23. FT-IR Spectrum of $\mathrm{Ni}(\mathrm{CO})_{3}\left(\mathrm{PFc}_{2} \mathrm{Ph}\right)(\mathbf{2 b})$ in $\mathrm{CH}_{2} \mathrm{Cl}_{2}$. 


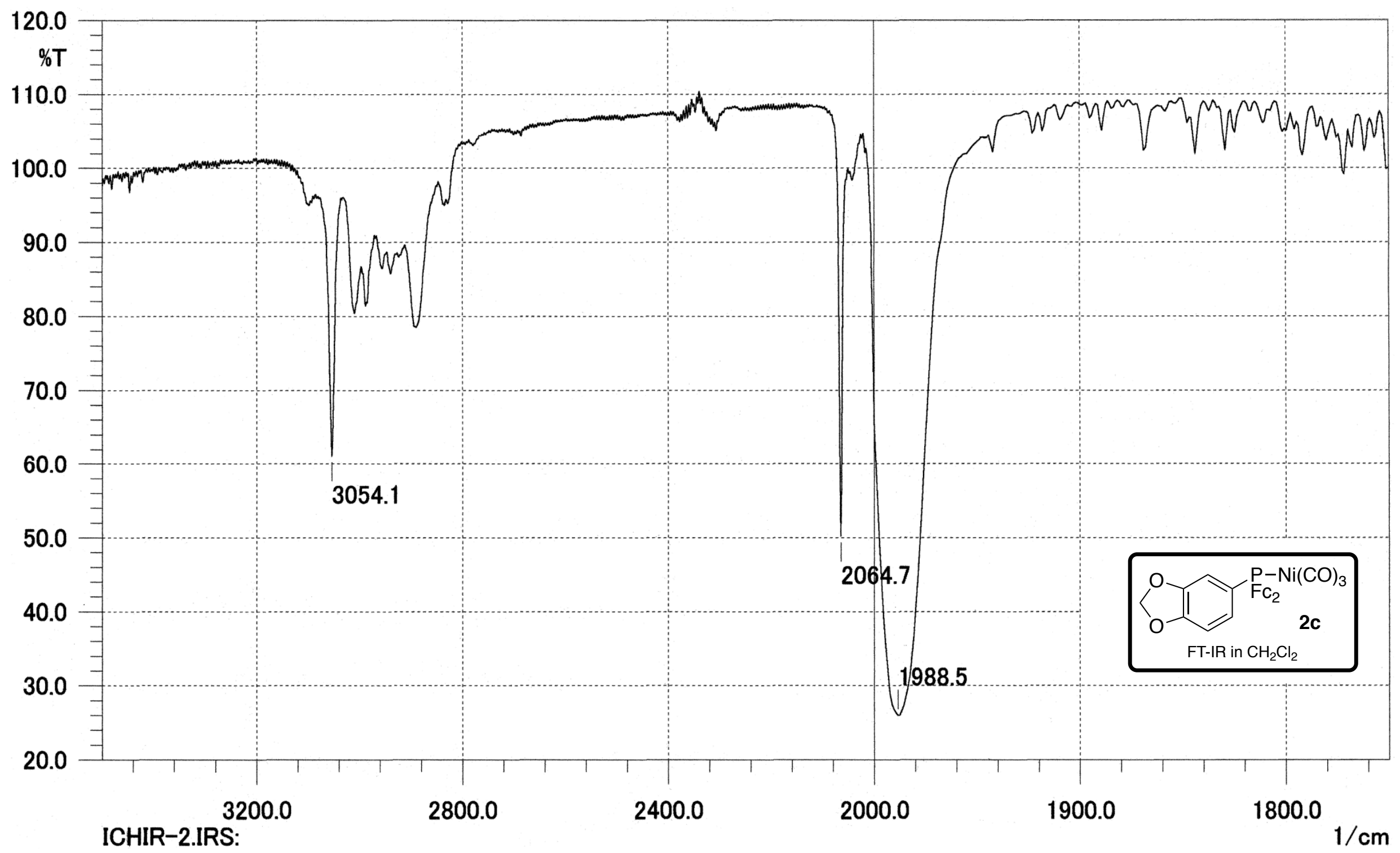

Figure S24. FT-IR Spectrum of $\mathrm{Ni}(\mathrm{CO})_{3}\left[\mathrm{PFc}_{2}\left(3,4-\mathrm{OCH}_{2} \mathrm{O}-\mathrm{C}_{6} \mathrm{H}_{3}\right)\right](\mathbf{2 c})$ in $\mathrm{CH}_{2} \mathrm{Cl}_{2}$. 


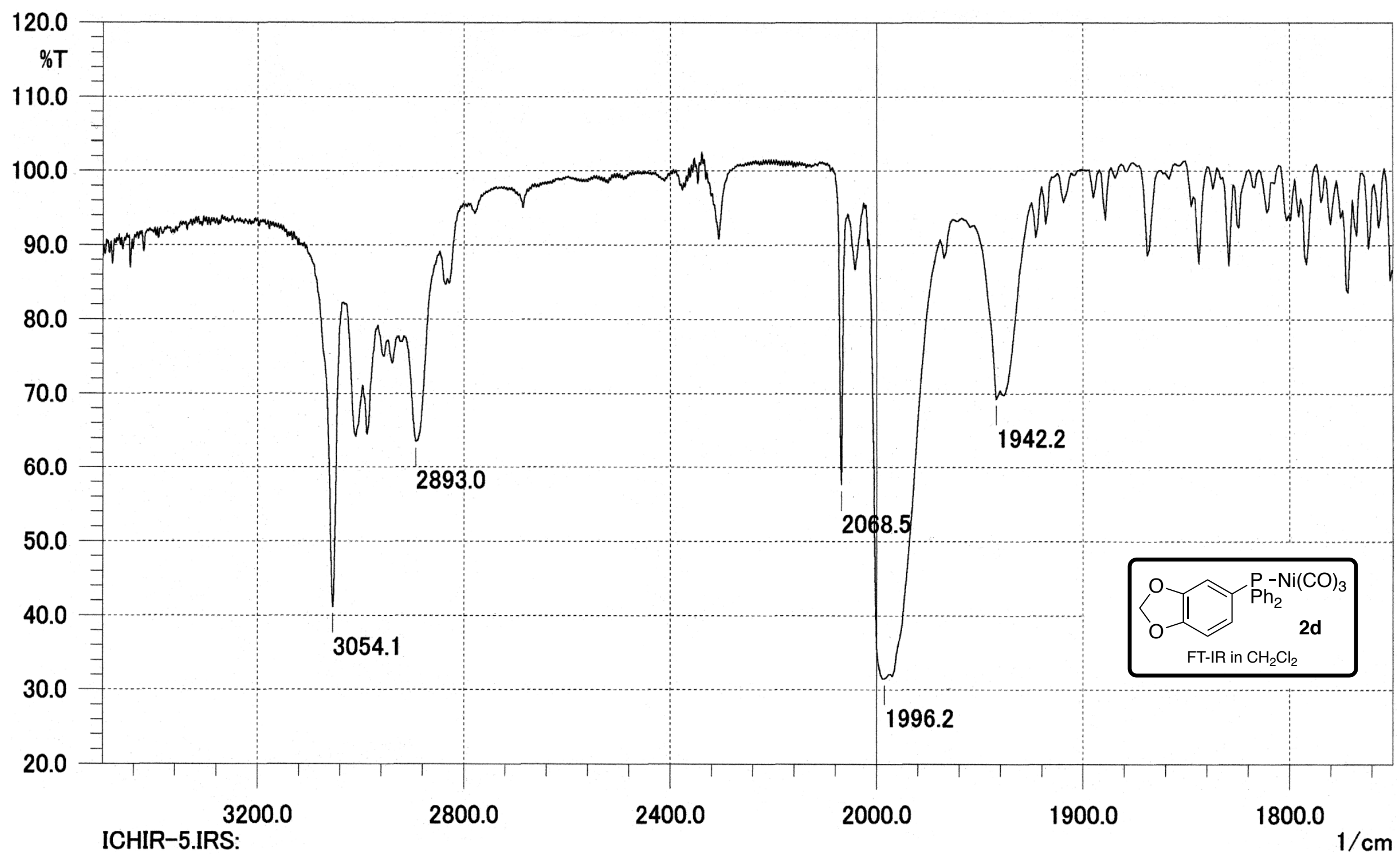

Figure S25. FT-IR Spectrum of $\mathrm{Ni}(\mathrm{CO})_{3}\left[\mathrm{PPh}_{2}\left(3,4-\mathrm{OCH}_{2} \mathrm{O}-\mathrm{C}_{6} \mathrm{H}_{3}\right)\right](\mathbf{2 c})$ in $\mathrm{CH}_{2} \mathrm{Cl}_{2}$. 